\title{
LTP: GluN2B on the go
}

\author{
Lucas Matt \& Johannes W Hell
}

\begin{abstract}
LTP, the lasting increase in synaptic transmission following heightened activity, is viewed as the physiological basis of learning. In this issue of The EMBO Journal, Dupuis et al find that certain NMDARs diffuse away upon LTP. Antibodies against the NMDAR from patients with autoimmune synaptic encephalitis prevent this redistribution and LTP.
\end{abstract}

See also: JP Dupuis et al (April 2014)

$\mathrm{D}$ uring basal neurotransmission, glutamate activates $\mathrm{Na}^{+}$influx through AMPA receptors (AMPARs) to depolarize postsynaptic sites. Bursts of highfrequency neurotransmission induce $\mathrm{Ca}^{2+}$ influx through NMDA receptors (NMDARs) to stimulate the $\mathrm{Ca}^{2+}$ and calmodulindependent kinase CaMKII (Lisman \& Hell, 2008; Nicoll \& Roche, 2013). These events can cause LTP, that is, permanently enhance the strength of a synapse, by increasing postsynaptic AMPAR content (Lisman \& Hell, 2008; Nicoll \& Roche, 2013).

Most AMPARs consist of two GluA1 and two GluA2 subunits and NMDARs of two GluN1 and two GluN2A or GluN2B subunits (Gray et al, 2011). PSD-95 anchors AMPAR and NMDAR at postsynaptic sites (Lisman \& Hell, 2008; Nicoll \& Roche, 2013). Dupuis et al (2014) now find that in immature neurons, lateral diffusion of GluN2B (but not GluN2A) increases $1-4$ min after LTP, which they induced by brief glycine application to temporarily increase NMDAR activity. The result is a reduction in postsynaptic GluN2B. These findings mirror electrophysiological work showing that in young (P2-9) rats, GluN2B is swiftly replaced by GluN2A following LTP (Bellone \& Nicoll, 2007). Dupuis et al now provide a cellular mechanism for this GluN2B/2A switch.
The GluN2B mobilization requires two kinases, CaMKII and the casein kinase CKII (Fig 1A). Activation of CaMKII upon $\mathrm{Ca}^{2+}$ influx leads to its autophosphorylation on T286. The dodecameric CaMKII then binds simultaneously to GluN2B and CKII linking the two together (Sanz-Clemente et al, 2013). CKII subsequently phosphorylates S1480 in the C-terminal SXV motif of GluN2B to disrupt its binding to PSD-95, which otherwise docks GluN2B at postsynaptic sites, allowing GluN2B to diffuse away (Fig 1A, center and right). An alternative, perhaps parallel mechanism is suggested by the finding that the GluN2B mobilization (Dupuis et al, 2014) as well as the GluN2B/2A switch during synapse maturation (Matta et al, 2011) also requires the activity of mGluR5, a Gq protein-coupled metabotropic glutamate receptor that activates PKC. PKC can stimulate the tyrosine kinase Pyk2, which then stimulates the tyrosine kinase Src to augment NMDAR activity and to promote LTP (Fig 1B, center and right) (Bartos et al, 2010).

To define a functional role of this GluN2B mobilization, the authors impair NMDAR diffusion with antibodies against the extracellular N-termini of GluN1 or GluN2B plus secondary, cross-linking antibodies. This manipulation does not affect basal GluA1 mobility, synaptic GluA1 or GluN1 content or NMDAR-mediated mEPSCs. It does, however, prevent the glycine-induced increase in postsynaptic GluA1 and GluA2 content in cultured neurons and LTP induced by electric stimulation in acute hippocampal slices, suggesting that GluN2B diffusion plays a role in LTP.

Autoantibodies against the $\mathrm{N}$-terminus of GluN1 can cause autoimmune synaptic encephalitis, which impairs cognition and memory. Dupuis et al find that IgG from sera of such patients prevent NMDAR diffusion and glycine-induced LTP. This effect does not require secondary, cross-linking antibodies raising the question how these autoimmune antibodies do impair NMDAR diffusion or whether effects other than impaired diffusion are responsible for the LTP block. The antibodies could have structural effects potentially preventing metabotropic signaling by NMDARs, which occurs independent of ion flux apparently via conformational changes in NMDARs (Nabavi et al, 2013). Although the antibodies do not affect CaMKII binding to GluN2B under basal conditions, they prevent the accumulation of CaMKII in spines that is otherwise seen upon $\mathrm{Ca}^{2+}$ influx via NMDAR and requires binding of CaMKII to the $\mathrm{C}$-terminus of GluN2B, as needed for LTP (Halt et al, 2012). Lateral mobility of GluN2B might be necessary for capturing CaMKII and perhaps moving CaMKII to locations such as perisynaptic sites. However, it seems more likely that GluN2B and with it CaMKII diffusing away from the central postsynaptic sites would impair rather than promote LTP. Alternatively, the antibodies could prevent a conformational change in the $\mathrm{C}$-terminus upon glutamate binding to GluN2B, which in turn prevents CaMKII binding or phosphorylation by Src and with it coincidentally LTP and lateral diffusion (Fig 1, left panels). Such a conformational model would also address the issue that within the time frame of GluN2B diffusion of 1-4 min after LTP induction, NMDAR activation at least by evoked transmission is no longer required for the maintenance of LTP (Adesnik \& Nicoll, 2007). Further supporting allosteric mechanisms, only CaMKII binding to GluN2B, but not CaMKII activity, is required beyond initial induction of LTP for LTP to be stable (Sanhueza et al, 2011). It is thus quite conceivable that the NMDAR antibodies prevent LTP by 

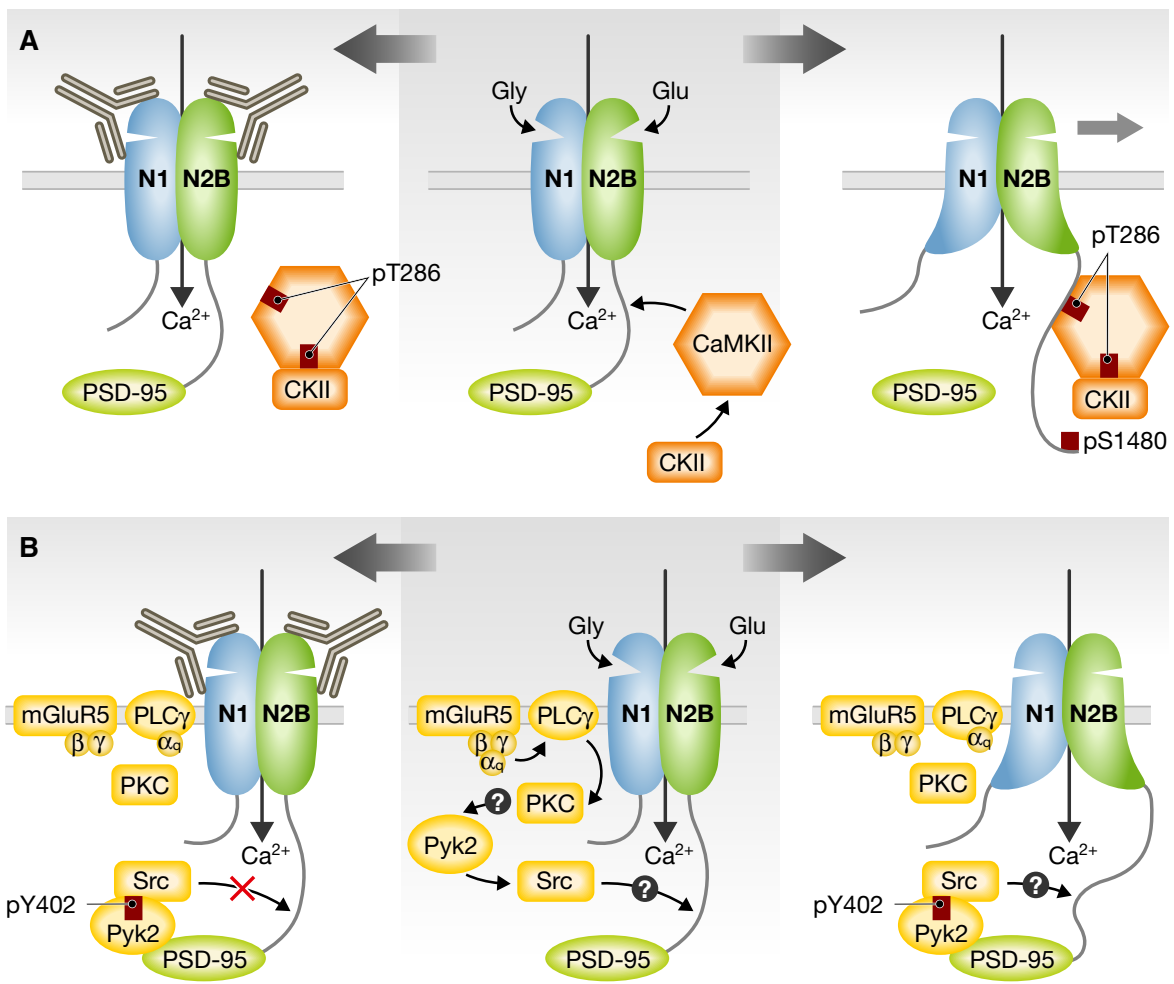

Figure 1. Hypothetical model of allosteric mechanisms upon binding of glutamate to GluN2B and the co-agonist glycine to GluN1.

(A) The $\mathrm{Ca}^{2+}$ influx triggers autophosphorylation of the dodecameric CaMKII on T286 (center) for the recruitment of CKII to GluN2B, S1480 phosphorylation, displacement of PSD-95, and GluN2B mobilization (right). (B) Activation of mGluR5 stimulates PKC, which, via unknown mechanisms, induces Pyk2 autophosphorylation on Y402. Src binds to PY402 and increases NMDAR activity via unknown mechanisms that might be important for the stabilization of LTP during its first 2-3 min (Bartos et al, 2010). In the models to the left, without affecting $\mathrm{Ca}^{2+}$ influx, antibodies are hypothesized to prevent a conformational change in the GluN2B C-terminus, which in turn prevents binding of the CaMKII/CKII couple (A) or regulation of NMDAR by Pyk2/SrC (B).

blocking the required activity-induced binding of CaMKII to GluN2B. Changes in postsynaptic GluN2B content could then be important during later phases of LTP and, more generally, synaptic development. Clearly, Dupuis et al raise many interesting open questions, which will inspire future work.

\section{Conflict of interest}

The authors declare that they have no conflict of interest.

\section{References}

Adesnik H, Nicoll RA (2007) Conservation of glutamate receptor 2-containing AMPA receptors during long-term potentiation. J Neurosci 27: 4598-4602

Bartos JA, Ulrich JD, Li H, Beazely MA, Chen Y, Macdonald JF, Hell JW (2010) Postsynaptic clustering and activation of Pyk2 by PSD-95. J Neurosci 30: 449-463

Bellone C, Nicoll RA (2007) Rapid bidirectional switching of synaptic NMDA receptors. Neuron 55: $779-785$
Dupuis JP, Ladépêche L, Seth H, Bard L, Varela J, Mikasova L, Bouchet D, Rogemond V, Honnorat J, Hanse E, Groc L (2014) Surface dynamics of GluN2B-NMDA receptors controls plasticity of maturing glutamate synapses. EMBO J 33: 842-861

Gray JA, Shi Y, Usui H, During MJ, Sakimura K, Nicoll RA (2011) Distinct modes of AMPA receptor suppression at developing synapses by GluN2A and GluN2B: single-cell NMDA receptor subunit deletion in vivo. Neuron 71 : $1085-1101$

Halt AR, Dallapiazza R, Yu H, Stein IS, Qian H, Junti S, Wojcik S, Brose N, Sliva A, Hell JW (2012) CaMKII binding to GluN2B is critical during memory consolidation. $E M B O$ / 31: 1203-1216

Lisman JE, Hell JW (2008) Long-term potentiation. In: Structural and Functional Organization of the Synapse, Hell JW, Ehlers MD (eds), pp. 501-534. Heidelberg: Springer

Matta JA, Ashby MC, Sanz-Clemente A, Roche KW, Isaac JT (2011) mGluR5 and NMDA receptors drive the experience- and activity-dependent NMDA receptor NR2B to NR2A subunit switch. Neuron 70 : 339-351

Nabavi S, Kessels HW, Alfonso S, Aow J, Fox R, Malinow R (2013) Metabotropic NMDA receptor function is required for NMDA receptor-dependent long-term depression. Proc Natl Acad Sci USA 110: 4027-4032

Nicoll RA, Roche KW (2013) Long-term potentiation: peeling the onion. Neuropharmacology 74: 18-22

Sanhueza M, Fernandez-Villalobos G, Stein IS, Kasumova G, Zhang P, Bayer KU, Otmakhov N, Hell JW, Lisman J (2011) Role of the CaMKII/NMDA receptor complex in the maintenance of synaptic strength. J Neurosci 31: $9170-9178$

Sanz-Clemente A, Gray JA, Ogilvie KA, Nicoll RA, Roche KW (2013) Activated CaMKII couples GluN2B and casein kinase 2 to control synaptic NMDA receptors. Cell Rep 3: $607-614$ 